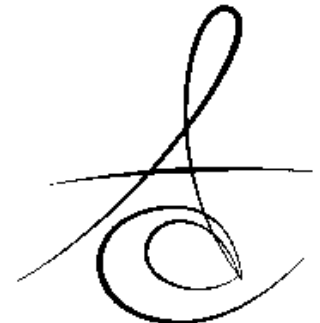

Makale Kodu/ArtIcle code: 1262

Makale Gönderilme tarihi: 14.08 .2013

Kabul Tarihi: 26.08.2013

\section{ÇOCUKLARDA DAMAK YARIĞI ONARIMI SONRASI GÖRÜLEN VELOFARİNGEAL YETMEZLÍK TEDAVİSİ}

\section{TREATMENT OF VELOPHARYNGEAL INSUFFICIENCY SEEN AFTER CLEFT PALATE REPAIR IN CHILDREN}

Prof. Dr. Ümit ERTAŞ*

\author{
Arş. Gör. Dt. Nesrin SARUHAN*
}

Yrd. Doç. Dr. Tevfik DEMİRCí**

\section{ÖZET}

Velofaringeal kompleks, posterior faringeal duvara doğru kapanarak oral ve nazal kaviteyi birbirinden ayırır. Velofaringeal yetmezlik (VFY) sıklıkla damak yarığı onarımı sonrasında görülür. Velofaringeal yetmezlik tedavisinde; Furlow palatoplastisi, faringeal flepler, Sfinkter faringoplastisi ve posterior faringeal duvar augmentasyonu gibi birçok cerrahi teknik kullanılmaktadır. Velofaringeal yetmezlik tedavisinde en sık kullanılan yöntemler; Sfinkter faringoplastisi ve faringeal fleplerdir. VFY'te kullanılan cerrahi tedaviler, velofaringeal mekanizmaya daha uygun yapı veya işlev oluşturmaya yöneliktir. Bu çalışmanın amacı, damak yarığı onarımı sonrasında görülen velofaringeal yetmezlik tedavisinde kullanılan cerrahi tekniklerin etkinliklerinin incelenmesidir.

Anahtar Kelimeler: Yarık damak, velofaringea yetmezlik, cerrahi prosedür

\section{ABSTRACT}

Velopharyngealcomplex separates the oral and nasal cavity closing towards the posterior pharyngeal wall. Velopharyngeal insufficiency (VFI) is often observed after cleft palate repair. Various surgical techniques including; Furlow palatoplasty, pharyngeal flap, Sphincter pharyngoplasty. and the posterior pharyngeal wall augmentation The most commonly used techniques are Furlow palatoplasty, pharyngeal flap, Sphincter pharyngoplasty and posterior pharyngeal wall augmentation. The surgical treatments of velopharyngeal insufficiency is aimed to form a more appropriate structure or function of velopharyngeal mechanism. The purpose of this study was to evaluate the efficiencies of the surgical techniques used for the treatment of velopharyngeal insufficiency seen after cleft palate repair.

Key Words: Cleft Palate, velopharyngeal insufficiency, surgical procedure

\section{GİRİŞ}

Velofarenks, oral ve nazal mukoza arasında bulunan musküler bir valvdir. Yumuşak damak ile lateral ve posterior faringeal duvarlardan oluşur. ${ }^{1}$ Velofaringeal kompleks, oral ve nazal kaviteyi birbirinden ayırır, yetersizliği sonucu da hipernazal konuşma görülür. Velofaringeal yetmezlik (VFY) genellikle yarık damak cerrahisi sonrasında görülmektedir. ${ }^{2}$ Özellikle izole submüköz damak yarığıyla ilişkilidir fakat farklı sendromlarla birlikte de görülebilmektedir. ${ }^{3}$ VFY, nöromusküler hastalık veya adenoidektomi sonrasında görülebildiği gibi, özellikle çocuklarda etiyolojisi tam olarak bilinmeyen durumlarda da görülebilmektedir. ${ }^{4}$ VFY, opere edilen yarık damaklı hastaların yaklaşık \% 20'sinde görülürken, opere edilmeyen submüköz yarık damaklı hastaların \% 10'unda görülmektedir. ${ }^{5}$ VFY, özellikle damak yarığı onarımı sonrasında görülür ve birçok vakada da cerrahi işlem gerekir. ${ }^{6}$

VFY'te kullanılan cerrahi tedaviler, velofaringeal mekanizmaya daha uygun yapı veya işlev oluşturmaya yöneliktir. Cerrahi tedavinin, hedefi uygun oronasal rezonansın sağlanması ve nazal kaçağın önlenmesidir. Uygulanan cerrahi yöntemler, velofaringeal valvde daralmaya bağlı nazal hava yolu obstrüksiyonu riski taşımaktadır. VFY tedavisinde birçok cerrahi teknik kullanılmaktadır. En sık kullanılan cerrahi teknikler, Furlow palatoplastisi, faringeal flepler, Sfinkter faringoplastisi ve posterior faringeal duvar augmentasyonudur. ${ }^{4}$

* Atatürk Üniversitesi Diş Hekimliği Fakültesi, , Ağız Diş ve Çene Cerrahisi Anabilim Dalı

** Atatürk Üniversitesi Diş Hekimliği Fakültesi, Pedodonti Anabilim Dalı 
Bu çalışmanın amacı, yarık damak ona- rımını takiben görülen VFY tedavisinde kullanılan cer- rahi prosedürlerin değerlendirilerek karşılaştııılmasıdır.

Velofaringeal yetmezlik tedavisinde en sik kullanılan cerrahi prosedürler, faringeal flep ve Sfinkter faringoplastisidir. Uzun yllar boyunca minimal VFY olan durumlarda en sık push-back veya uzatma teknikleri ile posterior duvar augmentasyonları kullanıldı. Günümüzde ise Furlow palatoplastisi başarı oranının yüksek olması nedeniyle daha kullanışı bir teknik olarak görülmekte, bu özelliklerinden dolayı, Furlow palatoplastisi özellikle minimal VFY olan ve solunum obstrüksiyonu riski bulunan durumlarda uygun bir tedavi yöntemi olarak kabul edilmektedir. ${ }^{3,7}$

Dailey ve ark. ${ }^{8}$ VFY'te faringeal flep ve Furlow palatoplastisinin konuşmadaki rezonans üzerine etkisini incelemişler ve her iki cerrahi tekniğin de hipernazaliteyi azaltmaya yardımcı olduğunu ve eşit oranda etkili olduğunu bulmuşlardır. Postoperatif değerlendirmede bu iki cerrahi prosedür arasında hipernazalite ve hiponazalite görülme oranı arasında herhangi bir fark bulunmadığını rapor etmişlerdir.

Faringeal flep cerrahisini takiben değişik oranlarda obstrüktif sleep apne görülebilmektedir. Orr ve ark. ${ }^{9}$ postoperatif 2. ve 3. günde $\% 90,3$. ayda ise $\%$ 20 , Sirois ve ark. ${ }^{10}$ postoperatif ilk 5 günde $\% 35$, Valnicek ve ark. ${ }^{11} \% 1.8$, Thurston ve ark. ${ }^{12}$ ise $\% 10$ oranında obstrüktif sleep apne görüldüğünü ve faringeal flep revizyonu gerektiğini bildirmişlerdir.

Wattanawong ve ark. ${ }^{13}$ velofaringeal cerrahide inferior ve superior bazlı faringeal fleplerin sonuçlarını karşılaştırdıkları çalışmalarında, inferior bazlı faringeal fleplerin daha başarıı olduğunu ve her iki grup arasında komplikasyon riski açısından istatistiksel olarak anlamlı bir fark bulunmadığını ifade etmişlerdir.

Pena ve ark. ${ }^{14} 88$ hastada faringeal flep cerrahisini takiben görülen perioperatif hava yolu komplikasyonlarını inceledikleri çalışmalarında, 7 hastada hava yolu obstrüksiyonu, 1 çocukta laringobronkospazm görüldüğü ve endotrakeal entübasyon yapıldığını, 1 hastada şiddetli obstrüktif sleep apne sonrası flep revizyonu gerektiğini, 3 hastada asistol ve apne görüldüğünü ve hastalardan birinin entübe edilmesine rağmen öldüğünü, 1 hastada ise nazal obstrüksiyon geliştiğini bildirmişlerdir.

Wang ve ark. ${ }^{15}$ velofaringeal yetmezliği bulunan ve faringoplasti uyguladıkları 219 hastada; tüm vakalarda hipernazalitede azalma, üfleme testinde çeşitli derecelerde azalma, konuşmadaki anlaşılabilir- likte, $\% 41^{\prime}$ den $\% 63$ 'e yükselme bildirmişler ve çalısmalarında, faringoplastinin velofaringeal yetmezlikte güvenli ve efektif bir yöntem olduğunu belirtmişlerdir.

Hirschberg yaptığı çalışmasında; faringeal fleplerdeki anatomik sonuçları başarılı bulmuştur. İmplant materyali uygulanarak yapılan augmentasyonlarda ise herhangi bir kayıp, absorpsiyon, migrasyon veya enfeksiyon bildirmemiştir. ${ }^{16}$

Serres ve ark. $^{4}$ Sfinkter faringoplastisi ile faringeal fleplerin sonuçlarını araştırmışlar ve 16 hastaya sfinkter faringoplastisi, 18 hastaya ise süperior bazlı faringeal flep uygulamışlardır. Faringoplastinin VFY'de daha yüksek oranda iyileşme ile sonuçlandığını tespit ettikleri bu çalışmalarında her iki teknikte de postoperatif olarak hiponazalite ve obstrüktif sleep apne, Sfinkter faringoplastisinde yüksek başarı oranı ve minimum obstrüktif sleep apne semptomları görüldüğünü ifade etmişlerdir.

Collins ve ark. ${ }^{5}$ VFY tedavisinde faringeal flep ile Sfinkter faringoplastisini karşılaştırdıkları çalışmalarında; faringeal flep prosedürünün daha başarıı olduğunu tespit etmişlerdir.

De Serres ve ark. ${ }^{4} 16$ hastaya Sfinkter faringoplastisi, 18 hastaya ise faringeal flep uygulaması sonucunda, faringoplasti uygulamalarında (\%50) faringeal fleplere (\%22) göre daha yüksek oranda VFY'de iyileşme görüldüğünü bildirmişlerdir.

Pensler ve ark. ${ }^{17} 75$ faringeal flep, 10 Sfinkter faringoplastisi uyguladıkları çalışmalarındada, her iki grupta da VFY'de iyileşme görüldüğünü belirtmişlerdir.

Witt ve ark. ${ }^{18}$ faringeal fleplerde \%20, Sfinkter faringoplastisinde ise \%16 oranında devam eden VFY'ye bağlı cerrahi revizyon gerektiğini ifade etmişlerdir.

Por ve ark. ${ }^{19}$ obstrüktif hava yolu semptomları nedeniyle faringeal flep revizyonunda; Furlow palatoplasti, faringeal flep ve faringeal fleple kombine Furlow palatoplastisi olmak üzere 3 farklı cerrahi prosedür uygulamışlar ve faringeal fleple kombine Furlow palatoplastisi uygulanan hastalarda, obstrüktif hava yolu semptomlarının iyileşmesinde istatistiksel olarak anlamlı farklııklar görüldüğünü belirtmişlerdir.

Sullivan ve ark. ${ }^{20}$ submükoz damak yarı̆ı̆ı ve VFY olan hastalarda 3 farklı cerrahi tekniğin konuşma üzerine olan etkilerini inceledikleri çalışmalarında, normal veya sınırda fonksiyonel birleşmenin faringeal flepte en yüksek oranda olduğunu bulmuşlardır. Ayrıca palatoplasti yapılan hastalarda başarının operasyon yaşından bağımsız olduğunu ve musküler retropozis- 
yonlandırmada, Furlow palatoplastisinin 2 flepli palatoplastiden daha etkili olduğunu bulmuşlardır.

Brigger ve ark. ${ }^{21} \mathrm{VFY}^{\prime}$ te kalsiyumhidroksiapatiti enjeksiyonla kullanmışlar ve hafif düzeyde VFY bulunan çocukların tedavisinde bu yöntemin yardımcı ve kullanışı olduğunu tespit etmişlerdir. Enjeksiyondan 24 aya kadar etkili ve güvenli olduğunu bildirmişlerdir. Bu prosedürün başarısında hasta seçiminin doğru yapılmasının çok önemli olduğunu vurgulamışlar ve bu tekniğin, çocuklarda hafif düzeyde VFY ve küçük velofaringeal boşlukların bulunduğu durumlarda tercih edildiğini ifade etmişlerdir. Kalsiyumhidroksiapatit ile posterior duvar augmentasyonundan, faringeal flep veya Sfinkter faringoplastisine bağlı morbiditenin görüldüğü durumlarda kaçınılması gerektiğini ifade etmişlerdir.

Lypka ve ark. ${ }^{22}$ posterior faringeal duvar augmentasyonu yapılan 111 hastada yaptıkları çalışmalarında; 12 hastadan, augmentasyon materyalinin çıkması sebebiyle, 1 hastadan ise postoperatif sleep apne nedeniyle implantın uzaklaştırıldığını rapor etmişlerdir. Uygulanan implantlarda başarı oranını \% 93 olarak bulmuşlardır. Augmentasyon yapılan 88 hastayı postoperatif konuşma açıından değerlendirmiş ve 64 hastada (\% 73) normal veya normale yakın konuşma, 22 hastada (\%25) orta düzeyde nazal konuşma ve 2 hastada (\%2) ise konuşmada düzelme görülmediğini belirtmişlerdir. Ayrıca, en yüksek çıkarıma oranın pürüzsüz yüzeyli silikon implantlarda görüldüğünü rapor etmişlerdir.

McComb ve ark. ${ }^{23}$ LeFort I maksiller ilerletme yapılan yarık damaklı hastalarda, yumuşak damak boyutunun operasyon sonrası VFY gelişme riskini artırdığını, bu nedenle VFY'de sıkıkla kullanılan faringeal flebin, LeFort I osteotomisi sonrası uygulanmasının en ideal zaman olduğunu bildirmişlerdir.

Paulson ve ark. $^{24}$ yaptıkları çalışmada; tonsillektomi yapılan hastalarda konuşmada anlaşııılık açısından preoperatif ve postoperatif olarak istatistiksel herhangi bir farklılık olmadığını bildirmişlerdir. ${ }^{24}$

Widdershoven ve ark. ${ }^{25}$ velo-kardio fasial sendromlu 25 hastada palatal uzatma cerrahisi sonrasında, konuşmada görülen değişiklikleri inceledikleri çalışmalarında, sadece 4 hastada ek cerrahi işlem gerektiğini, 15 hastada hipernazalitede azalma görülmediğini, 10 hastada ise iyileşme görüldüğünü fakat hiçbirinde normal konuşma sağlanamadığı bildirmişlerdir.

Abdel-Azziz, ${ }^{26}$ palatofaringeal ark ile bilateral superior konstrüktör kasın vertikal segmentinin diseksiyonunu ve sonrasında yumuşak damakta lateral insizyonlara yerleştirilerek superior konstrüktör kas ile palatofarengeus kasta beklenen sinerjik ve antagonist etkiyle velar harekette artmayı hedefleyen yeni bir prosedür tanımlamıştır. 17 olgunun 9'unda komple VFY eliminasyonu sağlanırken $\% 88$ oranında iyileşme görüldüğünü, bu iyileşmenin de orta düzeyli hipernazalitenin hafif, şiddetli hipernazalitenin ise orta düzeyli hale gelmesi şeklinde olduğu rapor etmiştir.

\section{SONUÇ}

VFY tedavisinde, çeşitli cerrahi yöntemler kullanılmakta olup bu yöntemlerle başarılı sonuçlar elde edilebilmektedir. En çok kullanılan ve başarı oranı daha yüksek olduğu düşünülen yöntemler ise faringeal flepler ve Sfinkter faringoplastisidir.

\section{KAYNAKLAR}

1. Premkumar S. Clinical application of palatal lift appliance in velopharyngeal incompetence. Journal of the Indian Society of Pedodontics and Preventive Dentistry 2011;29:S70-3.

2. Goudy S, Ingraham C, Canady J. Noncleft velopharyngeal insufficiency: etiology and need for surgical treatment. International journal of otolaryngology 2012; 2012: 296073.

3. Muntz HR. Current Techniques for Treatment of Velopharyngeal Insufficiency Operative Techniques In Otolaryngology-Head and Neck Surgery 2001;12:204-9.

4. de Serres LM, Deleyiannis FW, Eblen LE, Gruss JS, Richardson MA, Sie KC. Results with sphincter pharyngoplasty and pharyngeal flap. International journal of pediatric otorhinolaryngology 1999;48:17-25.

5. Collins J, Cheung K, Farrokhyar F, Strumas N. Pharyngeal flap versus sphincter pharyngoplasty for the treatment of velopharyngeal insufficiency: a meta-analysis. Journal of plastic, reconstructive \& aesthetic surgery : JPRAS 2012;65:864-8.

6. Koh KS, Kim SC, Oh TS. Management of velopharyngeal insufficiency using double opposing z-plasty in patients undergoing primary two-flap palatoplasty. Archives of plastic surgery 2013;40:97-103.

7. D'Antonio LL. Correction of velopharyngeal insufficiency using the Furlow double-opposing Zplasty. The Western journal of medicine 1997;167:101-2. 
8. Dailey SA, Karnell MP, Karnell LH, Canady JW. Comparison of resonance outcomes after pharyngeal flap and furlow double-opposing zplasty for surgical management of velopharyngeal incompetence. The Cleft palate-craniofacial journal : official publication of the American Cleft PalateCraniofacial Association 2006;43:38-43.

9. Orr WC, Levine NS, Buchanan RT. Effect of cleft palate repair and pharyngeal flap surgery on upper airway obstruction during sleep. Plastic and reconstructive surgery 1987;80:226-32.

10. Sirois M, Caouette-Laberge L, Spier S, Larocque $Y$, Egerszegi EP. Sleep apnea following a pharyngeal flap: a feared complication. Plastic and reconstructive surgery 1994;93:943-7.

11. Valnicek SM, Zuker RM, Halpern LM, Roy WL. Perioperative complications of superior pharyngeal flap surgery in children. Plastic and reconstructive surgery 1994;93:954-8.

12. Thurston JB, Larson DL, Shanks JC, Bennett JE, Parsons RW. Nasal obstruction as a complication of pharyngeal flap surgery. The Cleft palate journal 1980;17:148-54.

13. Wattanawong K, Tan YC, Lo L, Chen PK, Chen YR. Comparison of outcomes of velopharyngeal surgery between the inferiorly and superiorly based pharyngeal flaps. Chang Gung medical journal 2007;30:430-6.

14. Pena M, Choi S, Boyajian M, Zalzal G. Perioperative airway complications following pharyngeal flap palatoplasty. The Annals of otology, rhinology, and laryngology 2000;109:808-11.

15. Wang GM, Yang YS, Chen Y, Wu YL, Jiang LP, Liu Q. [Surgery for velopharyngeal insufficiency: a review of 219 modified pharyngoplasties]. Zhonghua er bi yan hou ke za zhi 2004;39:200-3.

16. Hirschberg J. Results and complications of 1104 surgeries for velopharyngeal insufficiency. ISRN otolaryngology 2012;2012:181202.

17. Pensler JM, Reich DS. A comparison of speech results after the pharyngeal flap and the dynamic sphincteroplasty procedures. Annals of plastic surgery $1991 ; 26: 441-3$.

18. Witt PD, D'Antonio LL. Velopharyngeal insufficiency and secondary palatal management. A new look at an old problem. Clinics in plastic surgery 1993;20:707-21.

19. Por YC, Tan YC, Chang FC, Chen PK. Revision of pharyngeal flaps causing obstructive airway symptoms: an analysis of treatment with three different techniques over 39 years. Journal of plastic, reconstructive $\&$ aesthetic surgery : JPRAS 2010;63:930-3.

20. Sullivan SR, Vasudavan S, Marrinan EM, Mulliken JB. Submucous cleft palate and velopharyngeal insufficiency: comparison of speech outcomes using three operative techniques by one surgeon. The Cleft palate-craniofacial journal : official publication of the American Cleft PalateCraniofacial Association 2011;48:561-70.

21. Brigger MT, Ashland JE, Hartnick CJ. Injection pharyngoplasty with calcium hydroxylapatite for velopharyngeal insufficiency: patient selection and technique. Archives of otolaryngology--head \& neck surgery 2010;136:666-70.

22. Lypka M, Bidros R, Rizvi M, Gaon M, Rubenstein A, Fox $D$, et al. Posterior pharyngeal augmentation in the treatment of velopharyngeal insufficiency: a 40-year experience. Annals of plastic surgery 2010;65:48-51.

23. McComb RW, Marrinan EM, Nuss RC, Labrie RA, Mulliken JB, Padwa BL. Predictors of velopharyngeal insufficiency after Le Fort I maxillary advancement in patients with cleft palate. Journal of oral and maxillofacial surgery : official journal of the American Association of Oral and Maxillofacial Surgeons 2011;69:2226-32.

24. Paulson LM, Macarthur CJ, Beaulieu KB, Brockman $\mathrm{JH}$, Milczuk HA. Speech outcomes after tonsillectomy in patients with known velopharyngeal insufficiency. International journal of otolaryngology 2012;2012: 912767.

25. Widdershoven JC, Stubenitsky BM, Breugem CC, MinkvanderMolen $A B$. Outcome of velopharyngoplasty in patients with velocardiofacial syndrome. Archives of otolaryngology--head \& neck surgery 2008;134:1159-64.

26. Abdel-Aziz M. Palatopharyngeal sling: a new technique in treatment of velopharyngeal insufficiency. International journal of pediatric otorhinolaryngology 2008;72:173-7.

\section{Yazışma Adresi:}

Nesrin Saruhan,

Atatürk Üniversitesi, Diş Hekimliği Fakültesi, Ağız Diş ve Çene Cerrahisi A.B.D.,

Erzurum, Turkiye

Tel.: 090442 2311747,

Fax:090 442 2360945,

E-mail:dt_nesrin@yahoo.com 